\title{
Clinical outcomes of antimicrobial lock solutions used in a treatment modality: a retrospective case series analysis
}

This article was published in the following Dove Press journal:

Clinical Pharmacology:Advances and Applications

15 June 2010

Number of times this article has been viewed

\author{
P Brandon Bookstaver ${ }^{1}$ \\ Katherine R Gerrald ${ }^{2}$ \\ Robert R Moran ${ }^{3}$ \\ 'South Carolina College of Pharmacy, \\ ${ }^{3}$ Health Science Research Core, \\ University of South Carolina, \\ Columbia, SC, USA; ${ }^{2}$ University of \\ North Carolina Hospitals, Chapel Hill, \\ NC, USA
}

Background: Antimicrobial lock therapy (ALT) may be considered as adjunctive therapy in the treatment of catheter-related bloodstream infections (CRBSI) when catheter removal is not a favorable option.

Objective: To evaluate the outcomes associated with ALT as adjunctive treatment of CRBSI.

Methods: This was a 24-month retrospective case series analysis evaluating patients treated for more than 24 hours with ALT. The primary outcome was blood culture sterilization for 30 days posttherapy. The impact of ALT duration and time to initiation on central venous catheter (CVC) salvage were evaluated. Logistic regression modeled the association between ALT and sterilization rates, with a prespecified level of significance $(\alpha)$ of 0.1 .

Results: Twenty-six cases were included in data analysis. Patients included ranged from 5 months to 82 years of age; $77 \%$ of patients were receiving total parenteral nutrition or chemotherapy. The majority of patients received vancomycin, daptomycin, or gentamicin combined with heparin in a lock solution. Blood culture sterilization was achieved in $69.2 \%$ of cases, and sterilization plus CVC retention was achieved in 11 cases $(42.3 \%)$. Longer durations of ALT ( $\geq 9$ days) were significantly correlated with blood culture sterilization (odds ratio $=1.367$, $P=0.077)$.

Conclusion: ALT used as an adjunct to systemic therapy for adequate duration in CRBSI can achieve CVC sterilization and retainment without subsequent infectious complications.

Keywords: antimicrobial lock therapy, central venous catheter, bacteremia, central lineassociated bloodstream infection

\section{Introduction}

Catheter-related bloodstream infections (CRBSI) are a primary concern in patients with long-term tunneled catheters, occurring in approximately $5 \%$ of the over 5 million central venous catheters (CVC) inserted annually. ${ }^{1,2}$ Significant morbidity and mortality may result with rates as high as $25 \%$ in high-risk patients with long-term CVC for hemodialysis, intravenous chemotherapy, and total parenteral nutrition (TPN). ${ }^{2}$ Gram-positive cocci are the most prevalent pathogens responsible for up to $70 \%$ of CRBSI, most commonly Staphylococcus aureus and coagulase-negative Staphylococci (CoNS). ${ }^{3}$ Removal of the catheter remains the primary intervention due to the formation of intraluminal biofilm.

Salvage therapy has been used, primarily in patients where catheter removal is not a viable option. This may be achieved by the instillation of a highly concentrated antimicrobial containing solution into the catheter lumen, otherwise known as antimicrobial
Correspondence: P Brandon Bookstaver South Carolina College of Pharmacy USC Campus, 7 I 5 Sumter Street, Columbia, South Carolina 29208, USA

$\mathrm{Tel}+\mathrm{I} 803-777-4786$

Fax +I 803-777-2820

Email bookstaver@sccp.sc.edu 
lock therapy (ALT). Concentrations of 100-1,000 times the minimum inhibitory concentration $\left(\mathrm{MIC}_{90}\right)$ of the planktonic bacteria may be required to achieve bioactivity against sessile bacteria within a biofilm. ${ }^{4}$ ALT is recommended by the Infectious Diseases Society of America (IDSA) Guidelines for management of CRBSI as an adjunct therapy to systemic antibiotics when the catheter is retained. ${ }^{1} \mathrm{~A}$ recent report from the Emerging Infections Network (EIN) of the IDSA demonstrated that approximately $37 \%$ of respondents use ALT in a treatment modality for catheter salvage. ${ }^{5}$ The overwhelming majority of clinicians (75\%-86\%) use vancomycin as their antibiotic of choice for CRBSI secondary to gram-positive pathogens. ${ }^{5}$ Published data on clinical outcomes in a treatment modality, as opposed to prophylactic use of ALT, is limited. Combined with systemic antibiotics, ALT has been shown to produce a clinical cure and result in catheter salvage in nearly $40 \%-100 \%$ of patients. ${ }^{6-9}$ Overall, better clinical outcomes are observed in CRBSI secondary to CoNS and gram-negative bacilli. , $^{1,-9}$

This case series analysis details experience with antimicrobial-anticoagulant solutions used as adjunctive therapy to systemic antibiotics in the treatment of CRBSI in patients with long-term CVC for hemodialysis, chemotherapy, and TPN. Included in this analysis are antimicrobial lock solutions with no previously reported clinical outcomes data.

\section{Patients and methods}

This was a retrospective case series analysis conducted at a single, university teaching hospital. Institutional review board approval was granted prior to data collection and presentation. Patients who received ALT over a 30-month period from May 2005 to November 2007 were screened for study inclusion. Cases were included if the patient received ALT for more than 24 hours in a treatment modality of an established CRBSI. Diagnosis of a CRBSI was based on clinical and microbiological evidence as defined by the National Patient Safety Network and Centers for Disease Control and Prevention and monitored by the institution's infection control staff. ${ }^{3}$ Cases were excluded if the patient had negative blood cultures before beginning ALT or if ALT was used less than 24 hours. In patients who received multiple courses of ALT, each course was documented as a separate case if it was deemed a new CRBSI. Infections were defined as a relapse if positive cultures from the CVC and/or peripheral blood resulted in the same organism within 30 days of treatment.

At this institution, nurses administering the ALT are instructed to maintain a locked lumen whenever the CVC is not in use; additional syringes of the lock solution are supplied by pharmacy upon request. In cases of dual-lumen CVC, a single lumen is locked for 24 hours continuously, whereas the other lumen is used for patient access. This process is alternated every other day. In patients receiving hemodialysis through a CVC, lumens are locked between dialysis sessions, for up to 72 hours continuously. Nurses routinely flush the lock solutions, unless the patient is not receiving the same agent systemically; the nurses are instructed to aspirate the solution in these cases.

The primary objective was to assess the percent of sterilized blood cultures from treatment with ALT. Primary endpoints were defined as negative blood cultures from both peripheral and CVC draws for the identified CRBSI pathogen for 30 days after end of ALT and systemic therapy. The secondary endpoints were CVC retention, infectious sequelae, and resolution of clinical signs and symptoms of infection. Host, patient, and treatment factors (eg, duration of ALT, concurrent systemic antibiotics, time to initiation of ALT, antibiotic used in ALT solution, causative organism) were examined for association with success of ALT. Performance of specific antimicrobial lock solutions and pathogen-specific outcomes were also analyzed. Data were collected to include age, gender, weight, indication for CVC, concurrent systemic antibiotic therapy, white blood cell (WBC) count, C-reactive protein (CRP), temperature curve, and all culture and susceptibility data during and for 30 days after conclusion of antibiotic lock and systemic antibiotic therapy for the defined CRBSI. All data regarding the lock solutions were collected to include concentrations of antimicrobials and anticoagulants, duration, and dwell time.

The data analysis used the most recent version of SAS (SAS Institute Inc, Cary, NC, USA). A multivariate logistic regression modeled the association between ALT and sterilization rates by estimating the odds ratio (OR). Significance was determined by the $P$-value of the associated Wald test compared with a 0.10 level of significance. The level of significance $(\alpha)$ was chosen to be 0.10 due to the small sample size. An OR greater than 1 indicates increased risk or positive association, whereas OR less than 1 indicates decreased risk or negative association. The following control/confounding variables were initially included in the model, but eventually dropped due to insignificance: age, weight, gender, reasons for CVC, pathogen, and specific lock solution received. Descriptive statistics were used to further define primary and secondary endpoint data.

\section{Results}

Over the 30-month study period, a total of 29 cases of ALT for CRBSI treatment were identified. Twenty-six cases were included in data analysis from 15 unique patients. One case 
was excluded because ALT was used for less than 24 hours, and 2 cases were excluded because the patients had negative blood cultures before beginning ALT. Patients included ranged from 5 months to 82 years of age. The breakdown for CVC placement in the 26 cases was as follows: TPN $(57.7 \%, \mathrm{n}=15)$, intravenous chemotherapy (Dnc; $23.1 \%$, $\mathrm{n}=6$ ), hemodialysis (HD; $15.4 \%, \mathrm{n}=4)$, and other long-term parenteral access $(3.8 \%, \mathrm{n}=1)$. The majority of cases $(62 \%)$ were pediatric patients less than 18 years of age. One patient (case \#23, Table 1) was neutropenic $\left(\mathrm{WBC}<500\right.$ cells $/ \mathrm{mm}^{3}$ ) at the initiation of ALT. No patients carried a diagnosis of HIV/AIDS. Table 1 provides a complete case analysis.

Antimicrobials used in lock solutions included $5 \mathrm{mg} / \mathrm{mL}$ or $10 \mathrm{mg} / \mathrm{mL}$ of vancomycin $(\mathrm{n}=8), 1 \mathrm{mg} / \mathrm{mL}$ of daptomycin $(\mathrm{n}=6), 2.5 \mathrm{mg} / \mathrm{mL}$ or $5 \mathrm{mg} / \mathrm{mL}$ of gentamicin $(\mathrm{n}=6)$, and $70 \%$ ethanol $(\mathrm{n}=1)$. In 5 cases, combinations of antimicrobial agents were used in lock solutions: vancomycin plus gentami$\operatorname{cin}(n=3)$ and vancomycin plus ethanol $(n=2)$. The majority of lock solutions $(24 / 26)$ were combined with heparin at variable concentrations ( 10 units $/ \mathrm{mL}$ up to 5,000 units $/ \mathrm{mL}$ ) depending on the indication for the CVC. Ethanol was not mixed with an anticoagulant in solution due to compatibility concerns. One patient received $5 \mathrm{mg} / \mathrm{mL}$ of vancomycin alone in solution without the addition of heparin (case \#18, Table 1). In the 2 patients receiving vancomycin plus ethanol locks, the agents were not combined in solution, rather the lumens were locked on alternating days with each individual antimicrobial in solution. No alternative anticoagulants were used in any of the lock solutions.

Concurrent systemic antibiotics were given in all cases, with the exception of 1 patient with an $\alpha$-hemolytic streptococcal infection, who received daptomycin lock therapy alone. In most cases $(22 / 26)$, the choice of agents for local lock therapy matched an agent in their concurrent systemic regimen. In the other 4 cases, 1 patient was receiving no systemic therapy, 1 patient was receiving an ethanol lock, and the other 2 patients were receiving gentamicin plus heparin lock therapy, while receiving alternative systemic $\beta$-lactams. In each case, the patient was receiving at least 1 active agent based on automated susceptibility profiles. The majority of causative organisms were gram-positive, with staphylococcal species being the most predominant. Of the 26 cases, $34.6 \%$ were CoNS, 19.2\% methicillin-resistant $S$. aureus (MRSA), 23.1\% gram-negative bacilli, 7.6\% Enterococcus species, $3.8 \%$ Streptococcus species, and $11.5 \%$ polymicrobial.

The primary end point of blood culture and CVC sterilization at 30 days after conclusion of systemic antibiotic therapy was achieved in $69.2 \%(n=18)$ of cases. Daptomycin and gentamicin achieved catheter sterilization in $100 \%$ of cases. When used as the sole antibiotic in solution $(n=8)$, vancomycin achieved a sterilization rate of $37.5 \%$. In all cases where daptomycin or vancomycin was used in a lock solution, these agents were also given as concurrent systemic therapy. Overall, culture sterilization rates in CRBSI caused by gram-positive pathogens were $80 \%$ for $S$. aureus, all of which were MRSA, and $77.7 \%$ for CoNS. One case of a polymicrobial infection secondary to CoNS and S. aureus resulted in sterilization failure and removal of the CVC. CRBSI secondary to gram-negative pathogens were sterilized at a rate of $83.3 \%$. A total of $13 \mathrm{CVC}$ cases $(50 \%)$ were salvaged with ABL therapy. In these 13 cases with $\mathrm{CVC}$ retainment, 1 patient experienced a recurrent CoNS bacteremia 2 months after the end of therapy (case \#20, Table 1). The patient ultimately expired; however, it was most likely attributed to progressive non-Hodgkin's lymphoma. Two of the $\mathrm{CVC}$ were retained despite persistent positive cultures in patients requiring continued hemodialysis. Eleven patients (42.3\%) achieved both blood culture sterilization and CVC salvage.

At the time of this case series analysis, the ALT dwell time was not documented at the institution; however, based on drug utilization records, the estimated mean dwell time in nonhemodialysis patients was 14 hours daily. In the cases of multilumen CVC, both lumens were locked with antimicrobial solution. Patients with a CVC in place for hemodialysis access received continuous dwells between sessions. The mean duration of ALT was 8.9 days. Longer ALT duration was significantly correlated with achieving catheter sterilization with an OR of 1.367 for each day above mean $(P=0.077)$. Of 10 patients who received at least 10 days of ALT, $9(90 \%)$ achieved the primary endpoint of sterilization. In respect to ALT initiation, $50 \%(n=13)$ of lock solutions were initiated within 48 hours of suspected CVC infection or initiation of systemic antimicrobials. Successful sterilization was achieved in $77 \%$ of these cases, although there was no significant correlation with day of ALT initiation $(\mathrm{OR}=1.034, P>0.1)$. There was no difference in outcomes based on number of lumens (single vs double) or type of catheter in place. No other host, pathogen, or treatment factors were significantly associated with increased sterilization rates.

Clinically, patients experienced defervescence an average of 42 hours after ALT initiation. In 1 case, the subject spiked a fever on day 10 of ALT and the CVC was subsequently removed. Data regarding WBC count and CRP were not reported due to inconsistent laboratory obtainment 


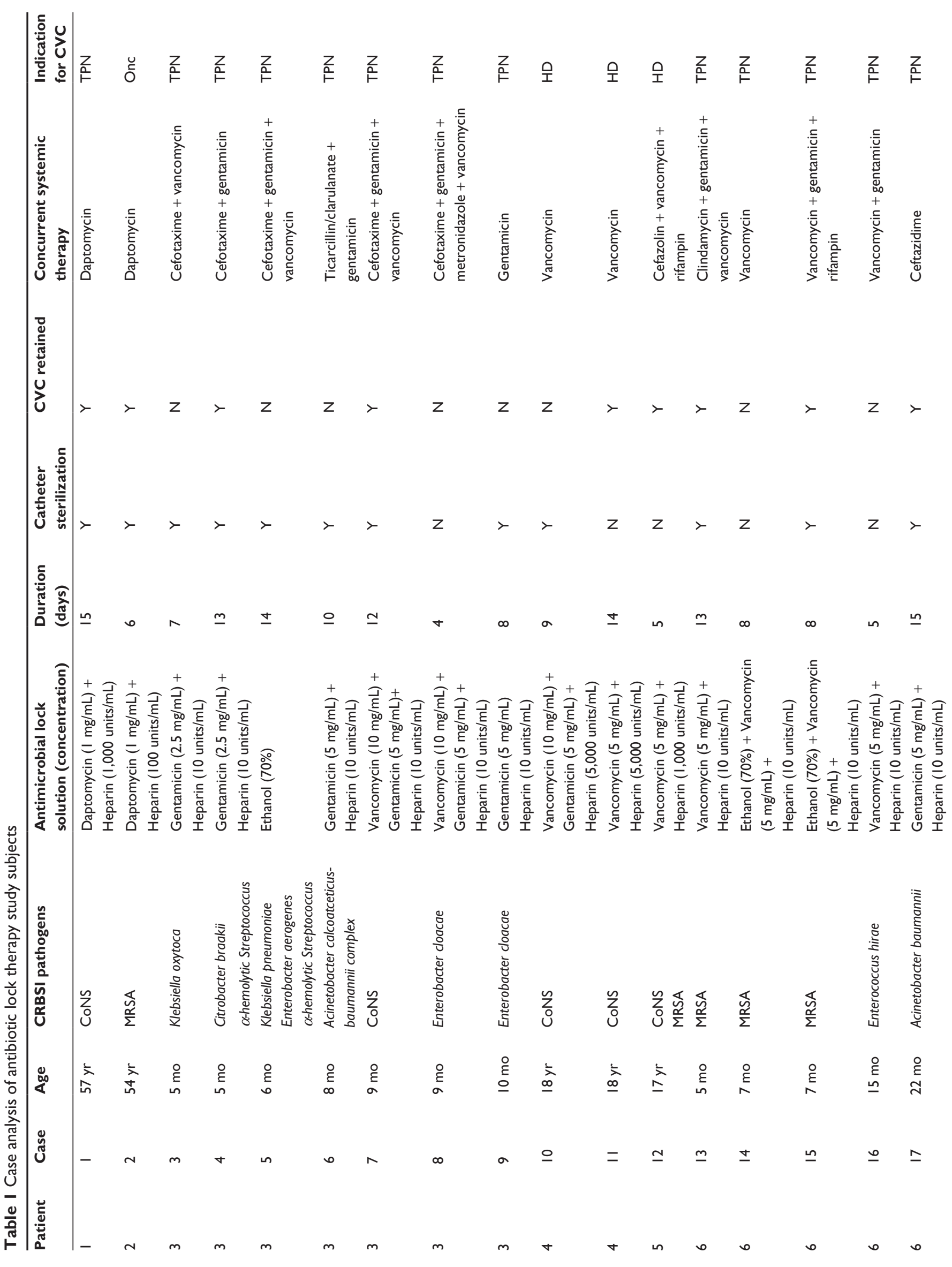




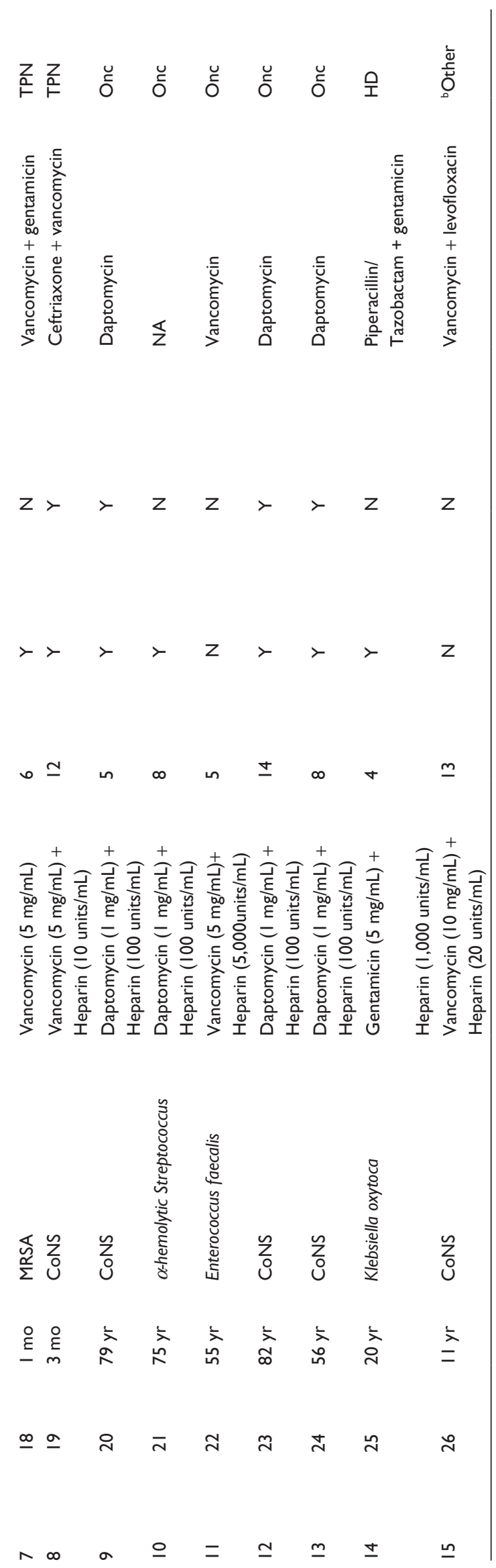

of these markers. One patient expired during the study period while receiving ALT. The catheter was retained; however, the death was determined unrelated to infectious complications.

\section{Discussion}

The use of long-term CVC is associated with a substantial risk of bloodstream infections. Although CVC removal is optimal, it is not always feasible in many patients who may have limited vascular access or high-risk surgical candidates. In our study, nearly $70 \%$ of patients successfully sterilized their blood with local ALT combined with systemic antibiotics, consistent with previous results. CVC removal was prevented in a total of 13 cases, and 11 of these patients had sustained negative cultures for the causative pathogen during the 30-day post-ALT period. Few randomized studies have been conducted investigating ALT in a treatment modality. Available data exists primarily from retrospective reports, open-label prospective studies, and case reports. Rjinders et $\mathrm{al}^{9}$ found that patients treated with ALT plus systemic therapy achieved a $67 \%$ cure rate compared with $43 \%$ cure rate in patients receiving only systemic antibiotics. Patients received either vancomycin or ceftazidime at a concentration of $500 \mathrm{mg} / \mathrm{L}$ plus heparin depending on the causative organism. Fortun et $\mathrm{al}^{10}{ }^{10}$ reported similar results ( $84 \%$ success rate) in a retrospective analysis of 29 episodes treated with ALT plus systemic antibiotics. Antibiotics used in that study included vancomycin, ciprofloxacin, or gentamicin lock solutions at $2 \mathrm{mg} / \mathrm{mL}$ plus heparin resulting in an $84 \%$ clinical and microbiological success rates. ${ }^{10}$ Additional studies have also produced similar rates of clinical and microbiological cure, including immunocompromised patients with neutropenia. ${ }^{11-14}$

In our study, duration of ALT was associated with a significant $(P=0.077)$ increased chance of success. Each additional day of therapy beyond 9 days (mean $=8.9$ days) of therapy resulted in a 1.367 times increased odds. IDSA guidelines for management of CRBSI suggest a targeted duration of 14 days. In a 2007 IDSA EIN antibiotic lock utilization report, $70 \%$ of respondents target 10-14 days for duration. ${ }^{5}$ Many prospective studies with successful outcomes used 14-21 days of ALT. ${ }^{6,79,11}$ A single study did demonstrate an $86 \%$ success rate against CRBSI primarily due to CoNS in an oncology population with only 3 days of ALT. ${ }^{13}$ Shorter durations, however, have been associated with treatment failures and attributable mortality from CRBSI. ${ }^{10}$ Onder et al ${ }^{15}$ demonstrated a significant improvement in treatment outcomes in pediatric patients on chronic hemodialysis when ALT was initiated early 
( $<72$ hours after suspected infection) vs late in the course of infection. ${ }^{15}$ In our study, ALT was initiated on average on day 3 of suspected CRBSI. There was no correlation to ALT success and day of initiation; however, a $77 \%$ sterilization rate resulted in patients when ALT was initiated $\leq 48$ hours into systemic therapy. Each of the 3 patients who received ALT on day 1 achieved sterilization and successful catheter salvage. Delay in consultation of infectious diseases pharmacists and physicians regarding the use of ALT and the inability to continue lock therapy following hospital discharge likely contribute to delayed initiation, shortened duration, and ultimately lower success rate. The available data supports the early initiation of ALT and continuation for a minimum of 14 days or for the complete duration of systemic therapy.

Vancomycin is the most studied antibiotic in both a treatment and prophylactic modality. ${ }^{6-12,16}$ In our case series, vancomycin was only successful in $37.5 \%$ of cases when used as the only antibiotic in solution. Vancomycin was also used in combination locks with both ethanol (vancomycin and ethanol locked separately on alternating days) and gentamicin (combined in solution) in 5 cases, with 2 reported failures. In vitro reports have suggested that vancomycin demonstrates less activity against biofilm-producing organisms and in a catheter model of infection compared with other agents, including minocycline, rifampin, and ciprofloxacin. ${ }^{17,18}$ An additional study demonstrated a more rapid elimination of $S$. epidermidis biofilms with gentamicin, linezolid, and eperezolid compared with $10 \mathrm{mg} / \mathrm{mL}$ of vancomycin. ${ }^{19}$ This lack of activity may be related to the delayed penetration of vancomycin into biofilm and decreased activity in the biofilm environment despite concentrations up to 1,000 times $\mathrm{MIC}_{90}$ values of planktonic bacteria. ${ }^{18}$ Systemic vancomycin was used concurrently in all cases where vancomycin was used in lock solution in our study. The average duration of vancomycin ALT was 9.12 days and lock therapy was initiated, on an average of day 3 of suspected CRBSI; no correlation to failure was noted. Although variable concentrations were used in this study, $5 \mathrm{mg} / \mathrm{mL}$ and $10 \mathrm{mg} / \mathrm{mL}$, there should be limited difference in treatment outcomes given the concentrations relative to MIC of the planktonic bacteria (at least 500 times the MIC). One major influence on the lack of comparable success of vancomycin ALT is 50\% of CRBSI were secondary to MRSA and enterococcal species, both known to have heightened virulence and lower eradication rates when treating biofilm-producing organisms compared with other pathogens, including CoNS. ${ }^{20-22}$
In this case series, lock solutions containing the single agents, daptomycin or gentamicin, achieved 100\% success in blood culture sterilization. Gentamicin locks were used only against gram-negative pathogens in our study, including 1 polymicrobial infection. Of note, success was achieved in both cases of multidrug-resistant Acinetobacter baumannii. Daptomycin was successfully used in 6 cases, 4 of which were CoNS and 1 case of MRSA. Daptomycin concentrations of $1 \mathrm{mg} / \mathrm{mL}$ were used at this institution. Addition of lactated Ringer's solution for calcium supplementation to reconstituted daptomycin is required for bioactivity. ${ }^{23}$ In vitro reports against biofilm-producing pathogens and data in a prophylactic modality support the utilization of both daptomycin and gentamicin in lock solutions. ${ }^{6,10,22-24}$ Ethanol as a $70 \%$ solution was also used in 3 cases in our study, twice in combination with vancomycin (vancomycin and ethanol locked separately on alternating days). The 1 pediatric patient who received daily ethanol locks did achieve success in this polymicrobial (Klebsiella pneumoniae plus Enterobacter aerogenes) CRBSI. When used on alternating days with vancomycin, ethanol locks achieved a 50\% (1/2) success rate. The failure was a CRBSI secondary to MRSA in a pediatric patient. Ethanol (70\%) has demonstrated success, 67\%-80\%, in additional prospective studies with limited number of patients. ${ }^{10,25}$ This high success rate was noted despite limited dwell times (4 hours/day) and shorter duration of therapy (5 days) in 1 study. ${ }^{10}$ A complete review of available literature regarding ethanol lock therapy has been recently published. ${ }^{26}$ Additional antimicrobials in lock solutions, including cefazolin, ceftazidime, and minocycline among others not included in our case series, have been investigated and may be viable options against targeted pathogens. ${ }^{1,9-11}$ Alternatives to heparin as an anticoagulant in solution, such as EDTA and citrate, have been used in combination with gentamicin, minocycline, and tigecycline. ${ }^{17,18,23,24,27-29}$ Given the effects of these metal ion chelators on biofilm integrity and synergism with other antimicrobials, they should be considered for use in lock therapy. ${ }^{17,18,30,31}$ These alternatives may also be viable options in patients unable to tolerate heparin in solution.

Our analysis was designed to describe clinical outcomes for several antimicrobial solutions against commonly encountered pathogens responsible for CRBSI. Inherent limitations for further analysis include the heterogeneous population, lack of consistency between responsible pathogens and treatment with specific lock solutions, variable concurrent systemic therapy, and inclusion of both pediatric and adult patients with variable indications for CVC. Because the administration and process of ALT was uniform, we were 
able to appropriately analyze the influence of specific host and technique factors, such as duration and time of ALT initiation. Although varied concentrations of antimicrobials were used in some cases (eg, vancomycin and gentamicin), each concentration remained at least 50 times the MIC of the planktonic bacteria and was typically in the 100-1,000 times range. Given these concentrations, we feel there would be little influence on the clinical outcomes between variable concentrations. Due to the small treatment population, no direct analysis was conducted between antimicrobial lock solutions. Our inability to accurately detect prolonged catheter survival did not allow us to report the number of catheter days saved. In addition, we were unable to report exact dwell times of the lock solutions; however, medication administration records were used to ensure lock solutions were allowed to dwell when the CVC was not in use and provide estimated times. Heparin was used as the anticoagulant in all lock solutions in our study, thus we were unable to report any results with antimicrobials alone or solutions containing alternative anticoagulants.

\section{Conclusion}

Patients who received ALT in combination with active systemic therapy for adequate duration achieved sterilization of blood and CVC cultures despite CVC retainment. Those who successfully retain the CVC should be carefully monitored for resolution of infection and development of infectious complications. In this study, 1 patient had a subsequent episode of bacteremia 2 months after conclusion of initial therapy, but no other sequelae were identified. CRBSI secondary to enterococcal species and $S$. aureus resulted in a higher failure rate, and careful risk-benefit should be weighed before initiating ALT in these patients. Given the lack of success with vancomycin ALT and previous data acknowledging limited effects on biofilm cells, it should be used with caution. In contrast to vancomycin, daptomycin and gentamicin lock solutions resulted in $100 \%$ success in achieving sterilization of blood and CVC. Selection of antimicrobial and anticoagulants in lock solutions should be made on bioactivity and clinical data against biofilm cells, available compatibility and stability information, and targeted at the likely pathogens responsible for CRBSI. Although CVC removal remains the treatment of choice, early initiation of ALT upon suspicion of CRBSI combined with protracted use for at least 2 weeks will increase the likelihood of successful treatment outcomes and should be considered a reasonable therapeutic alternative.

\section{Acknowledgments and disclosure}

Our sincere appreciation to Anne Hishon for her assistance with data collection and for the Palmetto Health Richland Pharmacy Department for aid with screening patients. P. Brandon Bookstaver receives research funding from Cubist Pharmaceuticals ${ }^{\circledR}$.

\section{References}

1. Mermel LA, Allon M, Bouza E, et al. Clinical practice guidelines for the diagnosis and management of intravascular catheter-related infection: 2009 update by the infectious diseases society of America. Clin Infect Dis. 2009;49:1249-1272.

2. Pittet D, Tarara D, Wenzel RP. Nosocomial bloodstream infection in critically ill patients: excess length of stay, extra costs, and attributable mortality. JAMA. 1994;271:1598-1601.

3. Bestul MB, VandenBussche HL. Antibiotic lock technique: review of the literature. Pharmacotherapy. 2005;25:211-227.

4. Pascual A, de Arellano ER, Martinez LM, Perea EJ. Effect of polyurethane catheters and bacterial biofilms on the in-vitro activity of antimicrobials against Staphylococcus epidermidis. J Hosp Infect. 1993;24:211-218.

5. Infectious Diseases Society of America Emerging Infections Network Report for Query. Antimicrobial lock prophylaxis and treatment of catheter-related bloodstream infections. http://www.int-med.uiowa. edu/research/ein/ALT_comments. Accessed December 10, 2008.

6. Krishnasami Z, Carlton D, Bimbo L, et al. Management of hemodialysis catheter-related bacteremia with an adjunctive antibiotic lock solution. Kidney Int. 2002;61:1136-1142.

7. Fernandez-Hildago N, Almirante B, Calleja R, et al. Antibiotic-lock therapy for long-term intravascular catheter-related bacteraemia: results on an open, non-comparative study. J Antimicrob Chemother. 2006;57:1172-1180.

8. Maya ID, Carlton D, Estrada E, Allon M. Treatment of dialysis catheterrelated Staphylococcus aureus bacteremia with an antibiotic lock: a quality improvement report. Am J Kidney Dis. 2007;50:289-295.

9. Rjinders BJ, Van Wijngaerden E, Vandecasteele SJ, Stas M, Peetermans WE. Treatment of long-term intravascular catheter-related bacteraemia with antibiotic lock: randomized, placebo-controlled trial. JAntimicrob Chemother. 2005;55:90-94.

10. Fortun J, Grill F, Martin-Davila P, Blazquez J, et al. Treatment of longterm intravascular catheter-related bacteraemia with antibiotic-lock therapy. J Antimicrob Chemother. 2006;58:816-821.

11. Poole CV, Carlton D, Bimbo L, Allon M. Treatment of catheter-related bacteraemia with an antibiotic lock protocol: effect of bacterial pathogen. Nephrol Dial Transplant. 2004;19:1237-1244.

12. Kim SH, Kang CI, Kim HB, et al. Outcomes of Hickman catheter salvage in febrile neutropenic cancer patients with Staphylococcus aureus bacteremia. Infect Control Hosp Epidemiol. 2003;24:897-904.

13. Sanchez-Munoz A, Aguado JM, Lopez-Martin A, et al. Usefulness of antibiotic-lock technique in management of oncology patients with uncomplicated bacteremia related to tunneled catheters. Eur J Clin Microbiol Infect Dis. 2005;24:291-293.

14. Broom J, Woods M, Allworth A, et al. Ethanol lock therapy to treat tunneled central venous catheter-associated blood stream infections: results from a prospective trial. Scand J Infect Dis. 2008;40:399-406.

15. Onder AM, Chandar J, Billings AA, et al. Comparison of early versus late use of antibiotic locks in the treatment of catheter-related bacteremia. Clin J Am Soc Nephrol. 2008;3:1048-1056.

16. Safdar N, Maki DG. Use of vancomycin-containing lock or flush solutions for prevention of bloodstream infection associated with central venous access devices: a meta-analysis of prospective, randomized trials. Clin Infect Dis. 2006;43:474-484.

17. Sherertz RJ, Boger MS, Collins CA, Mason L, Raad II. Comparative in vitro efficacies of various catheter lock solutions. Antimicrob Agents Chemother. 2006;50:1865-1868. 
18. Raad I, Hachem R, Tcholakian K, Sherertz R. Efficacy minocycline and EDTA lock solution in preventing catheter-related bacteremia, septic phlebitis, and endocarditis in rabbits. Antimicrob Agents Chemother. 2002;46:327-332.

19. Curtin J, Cormican M, Fleming G, Keelehan J, Colleran E. Linezolid compared with eperezolid, vancomycin, and gentamicin in an in vitro model of antimicrobial lock therapy for Staphylococcus epidermidis central venous catheter-related biofilm infections. Antimicrob Agents Chemother. 2003;47:3145-3148.

20. Mohamed JA, Huang DB. Biofilm formation by enterococci. J Med Microbiol. 2007;56(Pt 12):1581-1588.

21. Falagas ME, Kaspaskelis AM, Kouranos VD, Kakisi OK, Athanassa Z, Karageorgopoulos DE. Outcome of antimicrobial therapy in documented biofilm-associated infections: a review of the available clinical evidence. Drugs. 2009;69:1351-1361.

22. LaPlante KL, Mermel LA. In vitro activity of daptomycin and vancomycin lock solutions on staphylococcal biofilms in a central venous catheter model. Nephrol Dial Transplant. 2007;22:2239-2246.

23. Bookstaver PB, Williamson JC, Tucker BK, Raad II, Sherertz RJ. Activity of novel antibiotic lock solutions in a model against isolates of catheter-related bloodstream infections. Ann Pharmacother. 2009;43:210-219.

24. Raad I, Hanna H, Jiang Y, et al. Comparative activity of daptomycin, linezolid, and tigecycline against catheter-related methicillin-resistant Staphylococcus bacteremic isolates embedded in biofilm. Antimicrob Agents Chemother. 2007;51:1656-1660.
25. Dannenberg C, Bierbach U, Rothe A, Beer J, Korholz D. Ethanollock technique in the treatment of bloodstream infections in pediatric oncology patients with broviac catheter. J Ped Hematol Oncol. 2003; 25:616-621.

26. Maiefski M, Rupp ME, Hermsen ED. Ethanol lock technique: review of the literature. Infect Control Hosp Epidemiol. 2009;30:1096-1108.

27. Nori US, Manoharan A, Yee J, Besarab A. Comparison of lowdose gentamicin with minocycline as catheter lock solutions in the prevention of catheter-related bacteremia. Am J Kidney Dis. 2006; 48:596-605.

28. Bleyer AJ, Mason L, Russel G, Raad II, Sherertz R. A randomized, controlled trial of a new vascular catheter flush solution (minocyclineEDTA) in temporary hemodialysis access. Infect Control Hosp Epidemiol. 2005;26:520-524.

29. Dogra GK, Herson H, Hutchison B, et al. Prevention of tunneled hemodialysis catheter-related infections using catheter-restricted filling with gentamicin and citrate: a randomized controlled study. J Am Soc Nephrol. 2002;13:2133-2139.

30. Gray GW, Wilkinson SG. The effect of ethylenediaminetetra-acetic acid on the cell walls of some gram-negative bacteria. J Gen Microbiol. 1965; 39:385-399.

31. Banin E, Brady KM, Greenberg EP. Chelater-induced dispersal and killing of Pseudomonas aeruginosa cells in a biofilm. Appl Environ Microbiol. 2006;72:2064-2069.
Clinical Pharmacology: Advances and Applications

\section{Publish your work in this journal}

Clinical Pharmacology: Advances and Applications is an international, peer-reviewed, open access journal publishing original research, reports, reviews and commentaries on all areas of drug experience in humans. The manuscript management system is completely online and includes a very quick and fair peer-review system, which is all easy to use.

\section{Dovepress}

Visit http://www.dovepress.com/testimonials.php to read real quotes from published authors. 